\section{EMATS: An interactive computer program (APL) for generating ANOVA model equations and error terms}

\author{
WILLIAM T. McGOWAN III \\ Social and Behavioral Computer Sciences Laboratory \\ University of South Carolina, Columbia, South Carolina 29208
}

While the computational burden of analysis of variance (ANOVA) has been alleviated by the availability of the computer and statistical software packages, there remains the problem of determining the expected values of mean squares [E(MS)]. A determination of $\mathrm{E}(\mathrm{MS})$ is necessary in order to determine the appropriate error term for a particular independent effect (i.e., compute the $\mathrm{F}$ ratio). Although simple rules exist (Bennett \& Franklin, 1954; Cornfield \& Tukey, 1956) to derive the $\mathrm{E}(\mathrm{MS})$, the process is at times laborious and error prone.

The present paper describes an APL computer program that can be used to circumvent most, if not all, of these problems. Based on the design, the program derives the $E(M S)$ and the proper error term for a particular independent effect. Additionally, the program has the capability to form the model equation for the design. The program, called EMATS (expected means and test statements), offers to the consultant a means by which to "ease" his task and to the investigator a highly efficient way in which to "test" a particular

The author is indebted to Jerry Busemeyer, Social and Behavioral Computer Sciences Laboratory, and James Laughlin, Psychology Department, for critical readings of the manuscript. Thanks are also extended to Tricia Rickenbaker and Josie Rudolf for assistance in preparation of the manuscript. design. The EMATS APL program is written conversationally and is intended for users only vaguely familiar with APL. It is presented in Table 1 and explained below.

Function 1: EMATS. The purpose of this function is to organize program functions within a single function. To use EMATS, type the name of the function (EMATS), with its left and right arguments. The arguments may be formed in a number of different ways, but formed properly give the same results. The independent variables of the design form the left argument. Each independent variable is typed two characters in length, with one and only one space separating terms. The first character of each independent variable is always an $F$ or an $R$, representing fixed and random effects, respectively; the second, a unique character designator, represents the independent variable. $^{1}$ For example, for a design with four independent variables $(A=$ fixed, $B=$ fixed,$C=$ fixed, and $S=$ random effects), the left argument is typed as: 'FA FB FC RS'. There is no beginning or ending space and the character vector is enclosed within single quotes.

The relationship between independent variables form the right argument (e.g., crossed, nested). For a completely crossed design, type all character designators (independent variables) or an asterisk (*). For example, for a completely crossed design with four independent variables $(A, B, C, S)$, type the character vector as either (1) 'A B C S' or (2) '*'. In a design with nesting, the nested variable is followed immediately by parentheses that enclose the variable under which the variable is nested. For example, for a design with four independent variables ( $A, B, C, S$ ), with $B$ nested in $C$ and $S$ nested in $B$ and $C$, type as follows: ' $A B(C) S(B C)$ '. For illustra-

Table 1

EMATS Program Functions

Function 1: EMATS

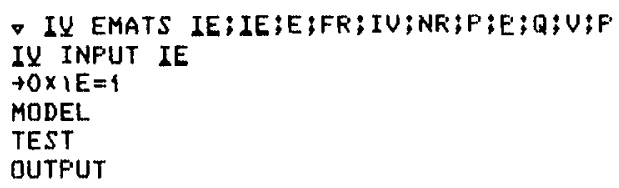

Function 2: INPUT

- IY INPUT IE;AIA;AO;AQ;C;DL;S;S1;S2:S1;S2

ErO

$D L+D B$

$S+F R(k)$ ? $\cdots+\cdots$

$C+(P+P+1) P S[Q]$

$A^{+}+1((-2 \uparrow(1 \phi A))=S[9]),\left(A+\left((-1+12) \phi\left((2 p S[9]) 0_{t}=A\right)\right)\right)$

$A O+{ }^{\prime}(((S 1++/(A \in S[3]))+(S 2++/(A \in S[5])))>0) / \cdots \rightarrow D L X \mid S\{\neq S 2 \cdots$

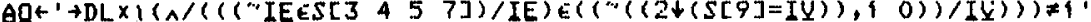

$=(S 1+(P A t, I Y)>1)>1+D 9 \times 1(* A) \neq 0)$

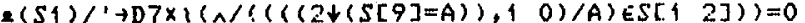

$=(S 2+(P A T, I E)>1) / \rightarrow \rightarrow D 9 \times 1(2 A) \geqslant 0$

Q $(S 2) / A A O^{\prime}$

$\mathrm{DL}+\mathrm{D} 10$ 
Table 1 Continued

Function 2 Continued

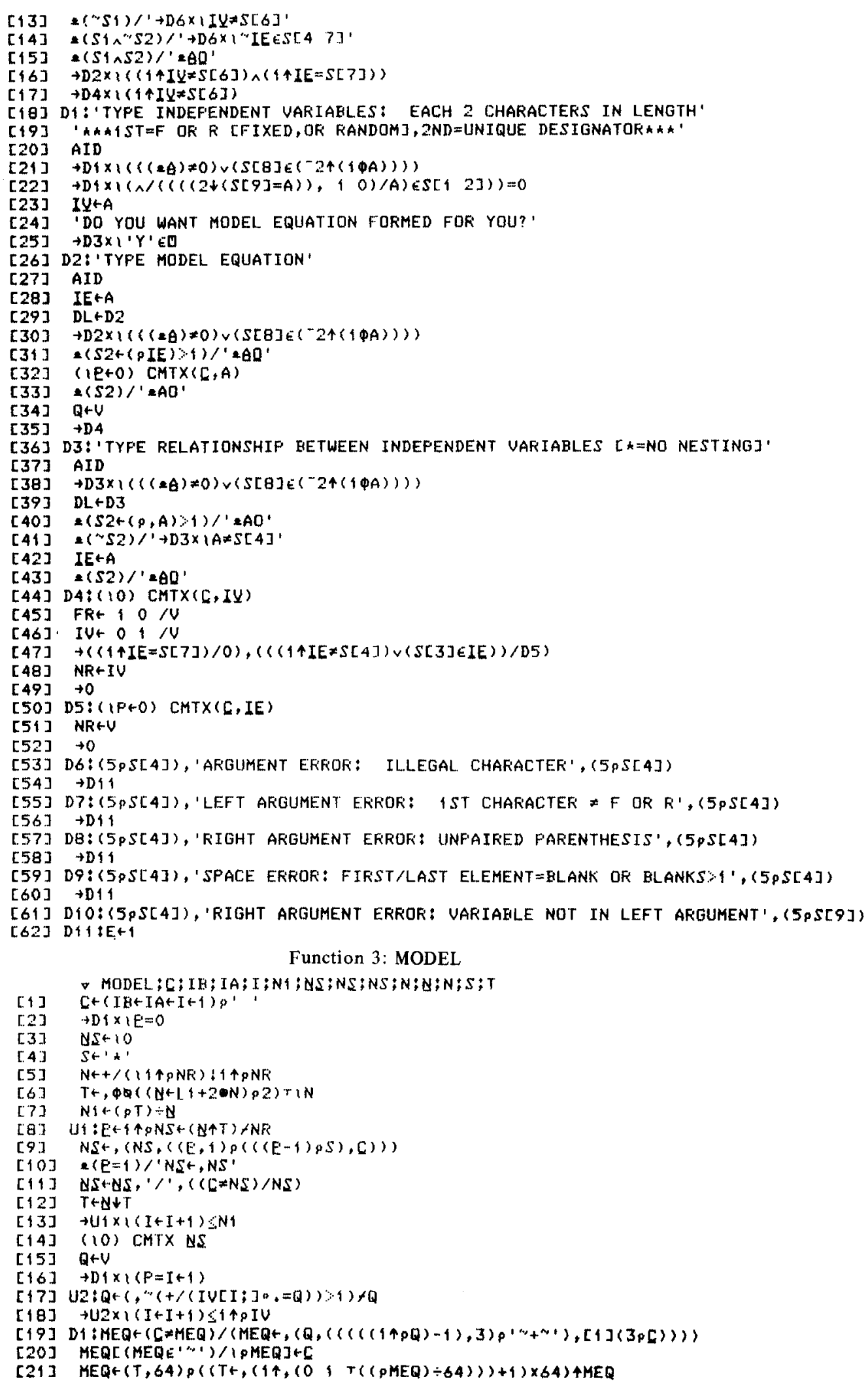


Table 1 Continued

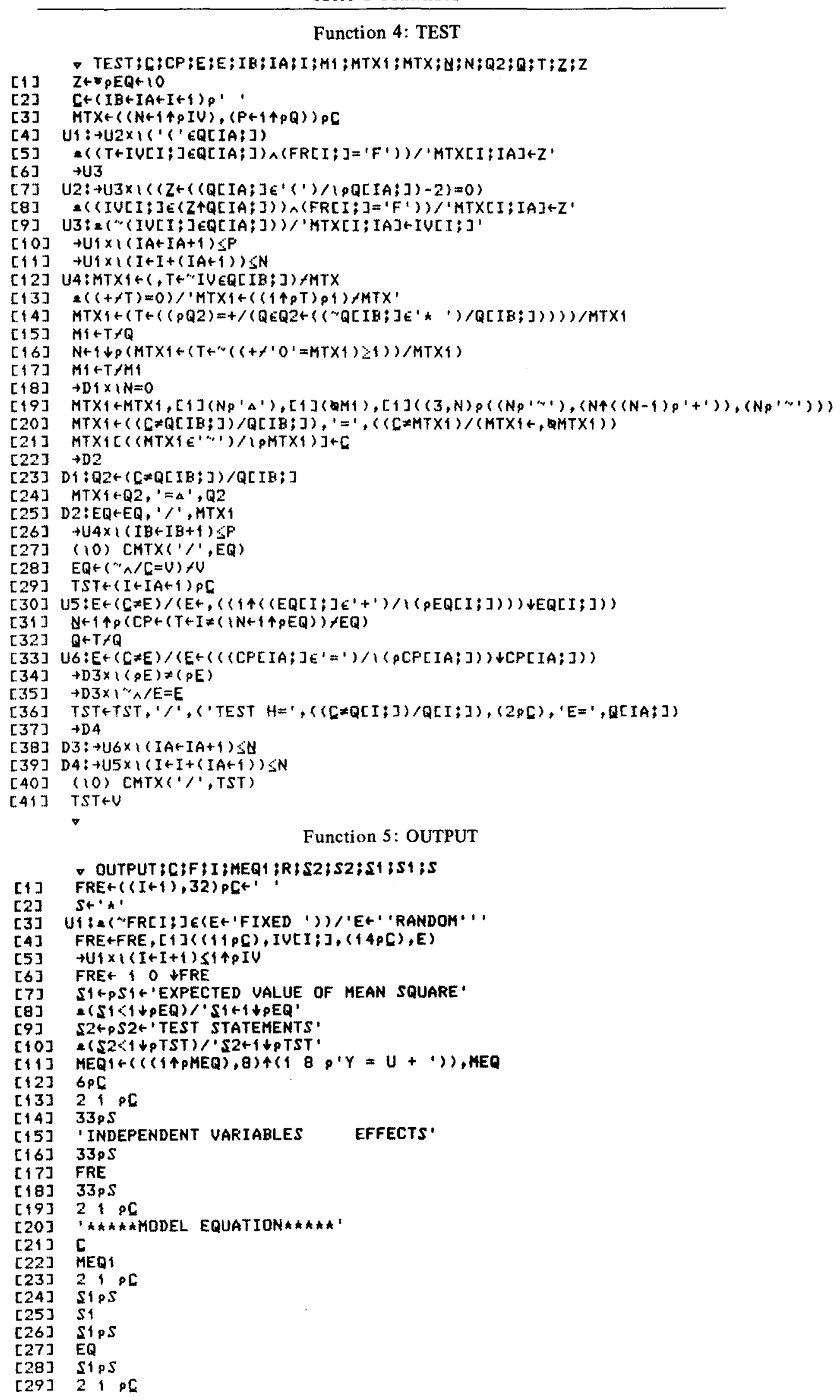


Table 1 Continued

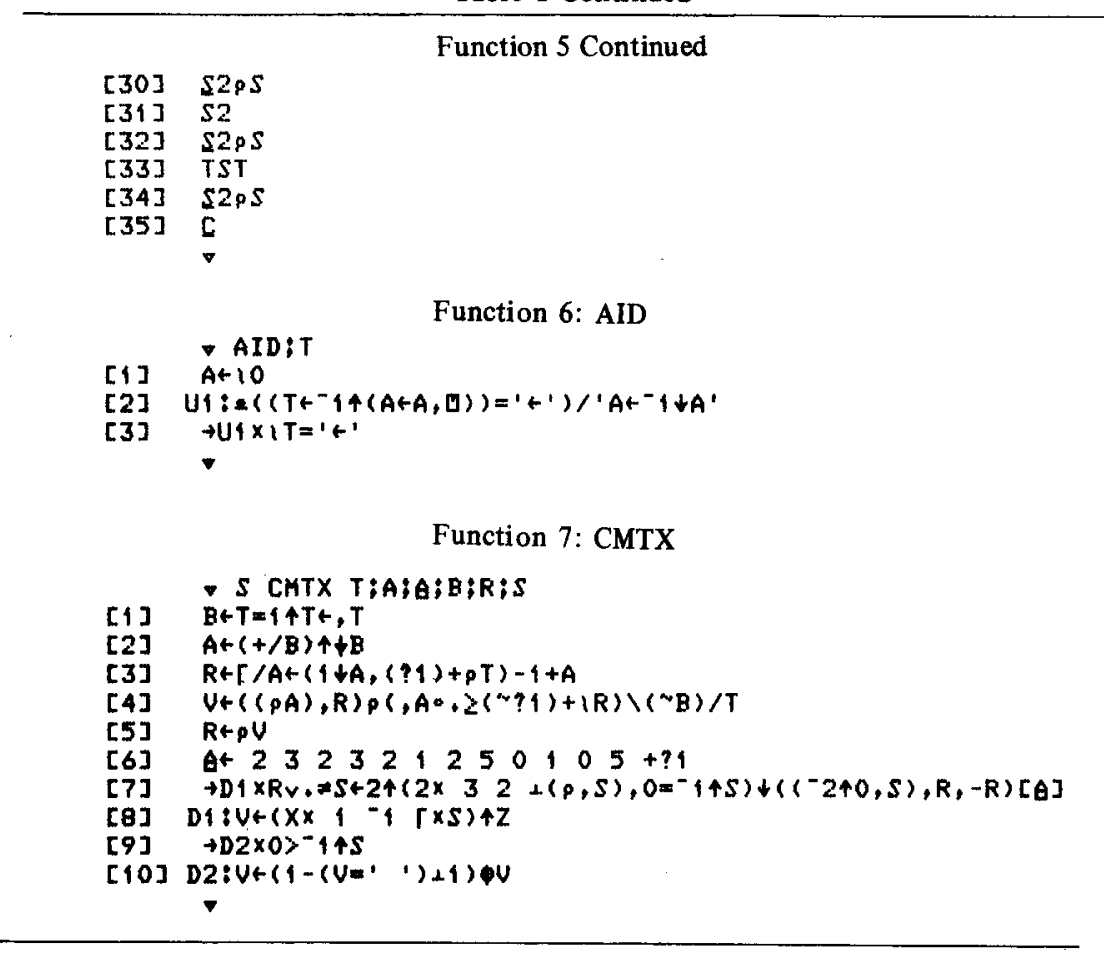

tions of output generated by such left-right arguments, see Examples 1 and 2 of Table 2.

If there is some question regarding the proper formation of left-right arguments, type a question mark (enclosed within single quotes) for the left argument and any APL character (enclosed within single quotes) for the right. Arguments typed in this manner result in the generation of queries within function INPUT (below) that request the appropriate information. In addition, the user may form his own model equation by typing the scaler monadic character not $(\sim)$ in single quotes for the right argument; the left argument is formed as described above. Left-right arguments, once formed, are evaluated within function INPUT (below). If either argument is not properly formed, an appropriate error message occurs and the program terminates. Errors evaluated include (1) any single illegal character argument, (2) character spacing error, (3) first character of each independent variable in left argument is not an $F$ or $R$, (4) unpaired parentheses in right argument, and (5) variable(s) in right argument not represented in left argument (see Example 3 in Table 2).

Function 2: INPUT. Function INPUT is designed to evaluate and integrate argument information. To use INPUT (if EMATS is not used), type the name of the function (INPUT) with its left-right arguments (INPUT arguments are the same as EMATS).

Queries (Qs), which are prompts for left-right argument design information, are generated within

Table 2

EMATS: Program Output

Example 1: (a) Crossed Design, (b) Argument Formation
'FA FE FC RS' EMATS ' $\star^{\prime}$

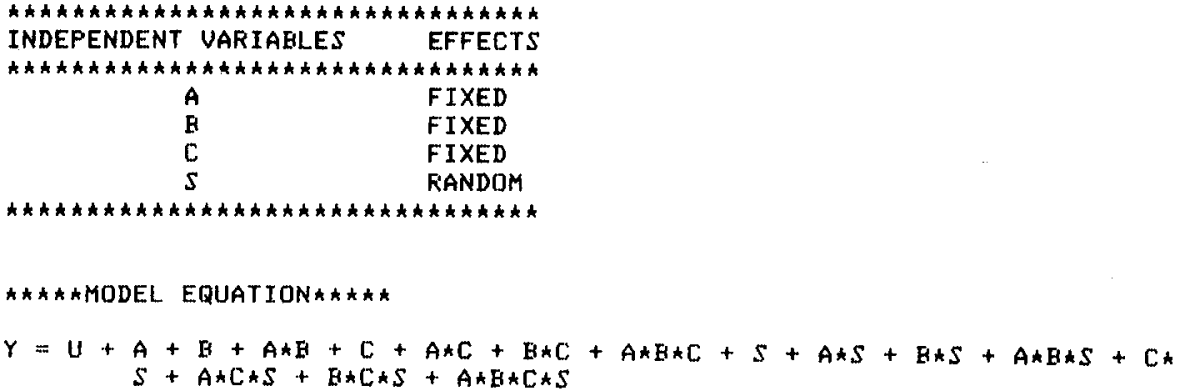


Table 2 Continued

Example 1 Continued

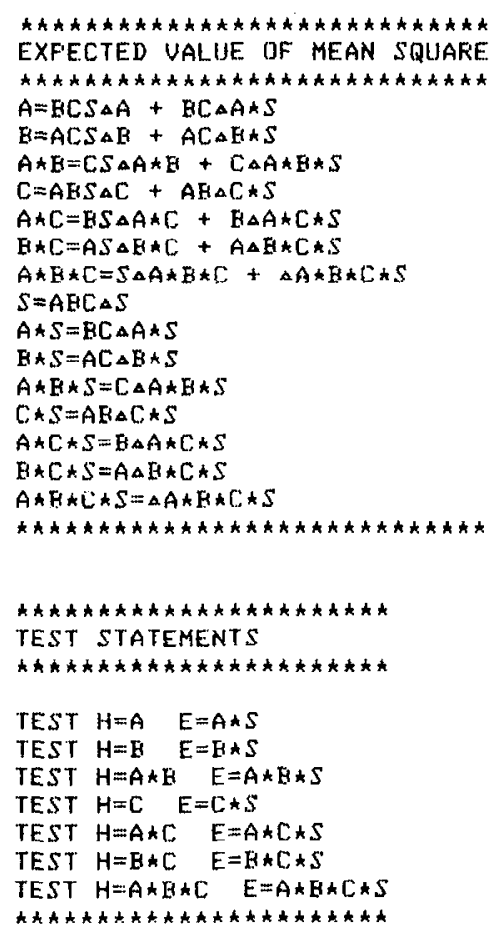

Example 2: (a) Hierarchical Design, (b) Argument Formation 'FA FE FC RS' EMATS 'A $B(A) C(A B)$ S (ABC)'

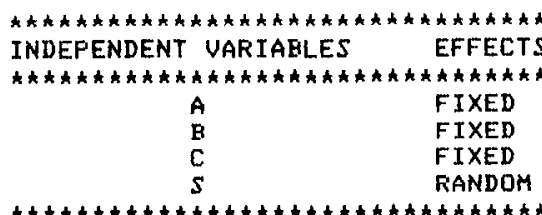

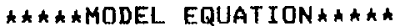

$Y=U+A+B(A)+C(A E)+S(A B C)$

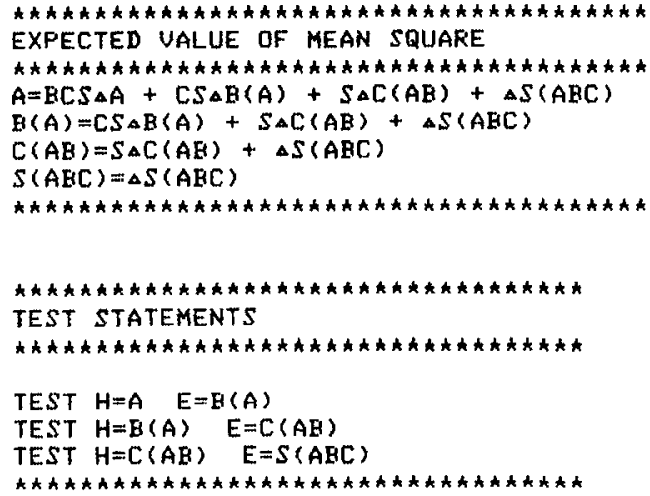

Example 3: Error Evaluation and Diagnostics

'E' EMATS 'A C B(A) S(ABC)' 
Table 2 Continued

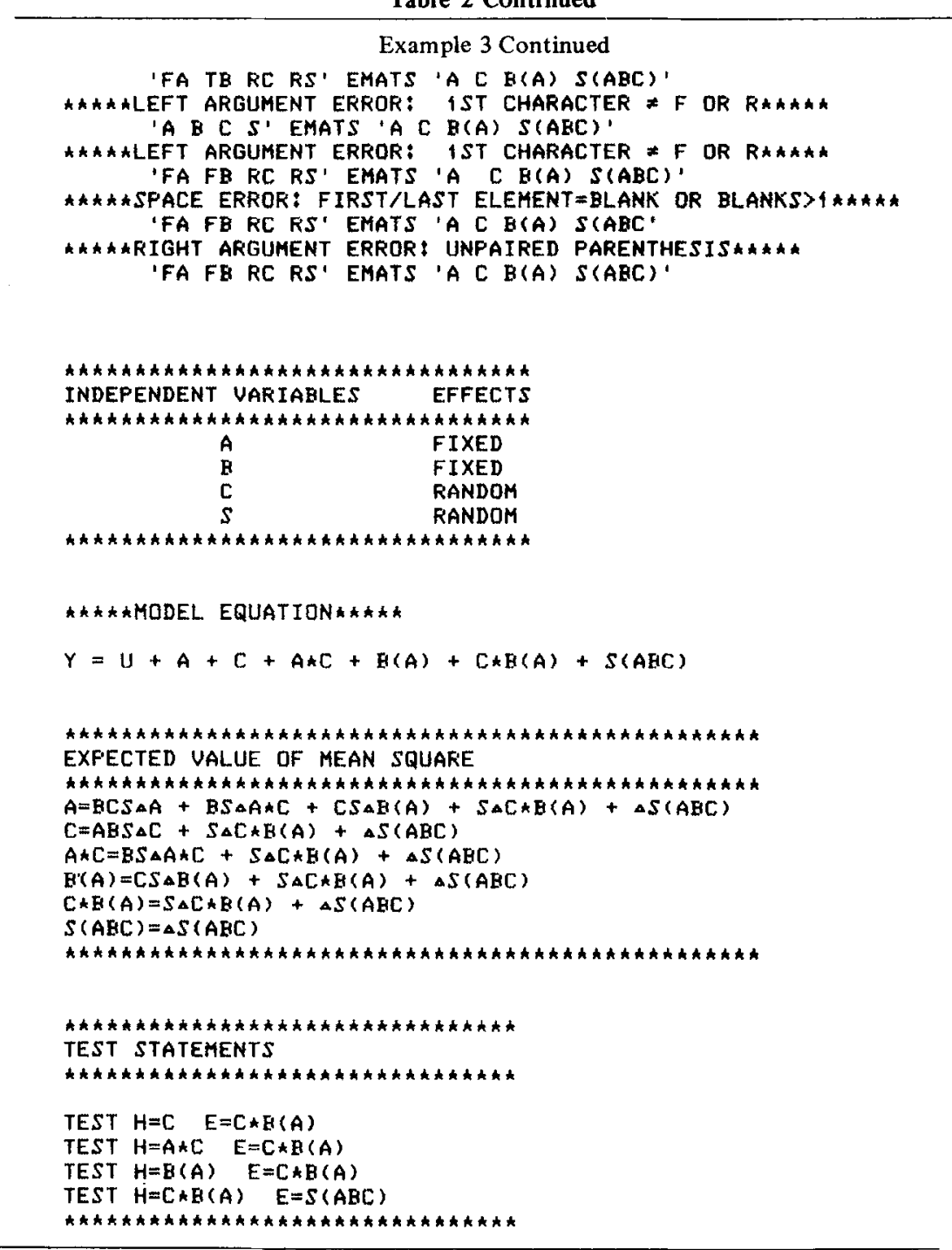

INPUT in response to a left argument question mark ('?) or a right argument not (' $\sim$ ), in which case only a single query (Q-3, below) is generated. Q-1 requests input of the multiple two-character independent vector. Q-2 requests whether or not internal (nonuser) model equation formation is desired. Q-3 occurs following a ' $\sim$ right argument or a negative response (NO or $N$ ) to Q-2 and requests input of the user-determined model equation. Q4, on the other hand, is generated after a positive response (YES or $\mathrm{Y}$ ) to $\mathrm{Q}-2$ and requests input of the relationship between independent variables. User responses to $Q s$ are the same as if no prompt had been requested (i.e., character information is typed in the same manner as that following left-right argument formation) except that single quotes are not used. If a response is not of the form required for design evaluation, a $\mathrm{Q}$ is repeated so that a response can be corrected. Errors evaluated are the same as that described above under function EMATS. Note that to branch out of a loop other than by a "correct" response to a $Q$, it is necessary to type the character-interrupt code [i.e., overstruck OUT (O, backspace, U, backspace, T)]. For illustrations of the use of INPUT, see Examples 1 and 2 in Table 3.

Function 3: MODEL. The model equation is formed within function MODEL based on design information from INPUT. To use MODEL, type the name of the function (MODEL). If EMATS is not used, it is first necessary to use INPUT. An example of variable (MEQ) output is presented in Example 2 in Table 3.

Function 4: TEST. The expected values of mean squares (stored under variable EQ) and test statements (stored under variable TST) are determined within function TEST. The format for the test statements is structured for implementation under SAS (Barr, Goodnight, Sall, \& Helwig, Note 1). TEST is based on an algorithm for determining E(MS) presented by Winer (1971, pp. 372-375). An example of variable (EQ, TST) output generated by function TEST is presented in Example 2 in Table 3.

Function 5: OUTPUT. Output from INPUT, MODEL, and TEST is organized by function OUTPUT. There are 
Table 3

Response Evaluation

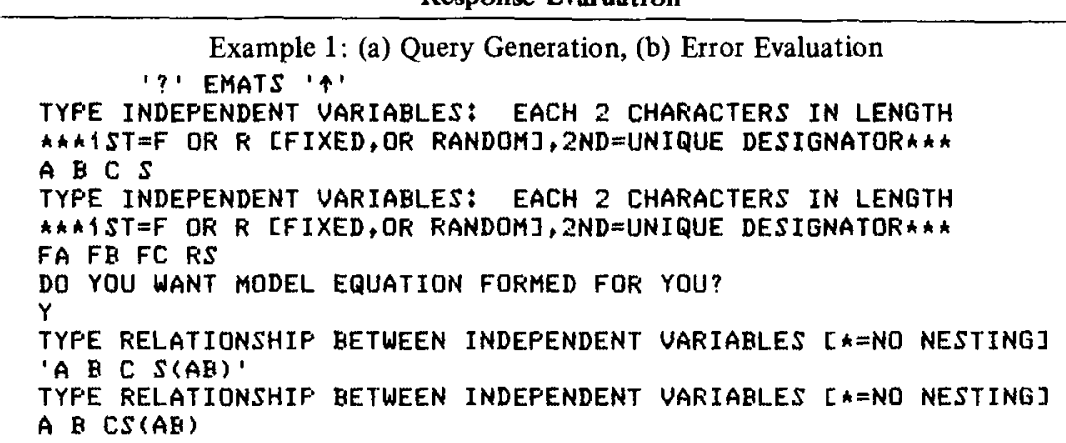

A $B \operatorname{Cs}(A E)$

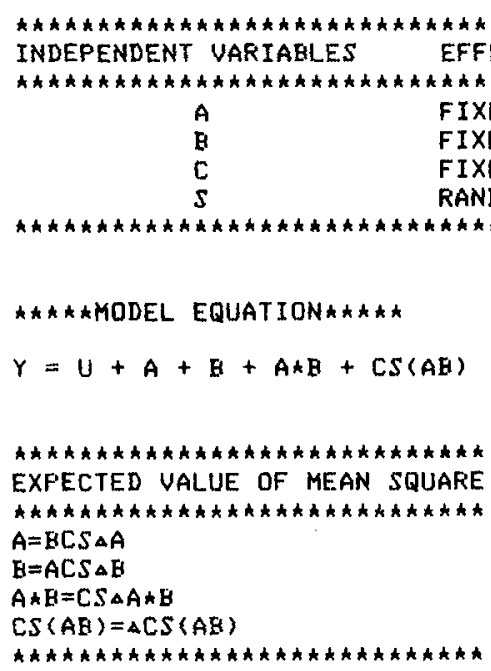

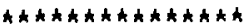

TEST STATEMENTS

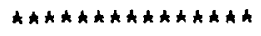

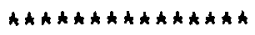

Example 2: (a) Sequential Function Use, (b) External Argument Formation Step 1: a) Left argument formation, b) function AID $\begin{array}{lll} & \text { AID } \\ \text { FA FB FC + } & \text { RD FE RS }\end{array}$

c) assignment of AID output

LEFT $+A$

Step 2: a) right argument formation, b) function AID

A $\operatorname{CS}_{S(A C)}^{\text {AID }}$

c) assignment of AID output

RIGHT+A

Step 3: a) Variable output (left argument) LEFT

FA FB FC RD FE RS

b) variable output (right argument) RIGHT

A B C DE $S(A C)$ 
Table 3 Continued

Example 2 Continued

Step 4: Function INPUT

LEFT INPUT RIGHT

Step 5: Function MODEL

MODEL

Step 6: Variable output (model equation)

MEQ

$A+B+A * B+C+A * C+B * C+A * B * C+D+A * D+B * D+A * B * D+C *$

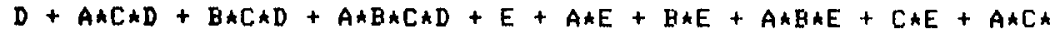

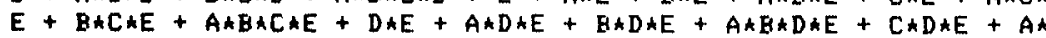

$C \star D \star E+B * C * D * E+A * B * C A D \star E+S(A C)+B A S(A C)+D * S(A C)+B * D * S($

$A C)+E * S(A C)+B \star E \star S(A C)+D * E \star S(A C)+E * D * E \star S(A C)$

Step 7: Function TEST

TEST

Step 8: Variable Output [E(MS)]

EQ

$A=B C D E S \triangle A+B C E S \triangle A A D+B D E A S(A C)+B E \triangle D+S(A C)$

$B=A C D E S \triangle B+A C E S A B * D+D E \triangle B * S(A C)+E \Delta B * D * S(A C)$

$A \star B=C D E S \triangle A \star B+C E S \triangle A \star B \star D+D E \triangle B * S(A C)+E \Delta F * D \star S(A C)$

$C=A B D E S A C+A B E S \triangle C * D+B D E \triangle S(A C)+B E \triangle D * S(A C)$

$A \star C=B D E S \triangle A A C+B E S \triangle A \star C A D+B D E \triangle S(A C)+B E \triangle D k S(A C)$

$B \star C=A D E S \triangle B * C+A E S \triangle B \star C * D+D E A B k S(A C)+E \triangle E * D * S(A C)$

$A \star B \star C=D E S \triangle A * B \star C+E S \triangle A \star B \star C * D+D E \Delta B * S(A C)+E \Delta B \star D \star S(A C)$

$D=A B C E S \triangle D+B E \triangle D \star S(A C)$

$A \star D=B C E S \triangle A * D+B E \triangle D \star S(A C)$

$B A D=A C E S \triangle B * D+E \triangle B * D A S(A C)$

$A \star B \star D=C E S \triangle A \star B \star D+E \triangle B \star D * S(A C)$

$C \star D=A B E S \triangle C \star D+B E \triangle D \star S(A C)$

$A \star C \star D=B E S \triangle A \star C \star D+B E \triangle D * S(A C)$

$E \star C \star D=A E S \triangle B \star C \star D+E A B \star D * S(A C)$

$A \star E \star C \star D=E S \triangle A \star B \star C \star D+E \triangle B \star D * S(A C)$

$E=A B C D S \triangle E+A B C S \triangle D * E+B D \triangle E \star S(A C)+B \triangle D \star E * S(A C)$

$A \star E=B C D S \triangle A \star E+B C S \triangle A \star D * E+B D A E \star S(A C)+B \triangle D * E * S(A C)$

$B \star E=A C D S \triangle B * E+A C S \triangle B \star D \star E+D \triangle B \star E \star S(A C)+\triangle B \star D * E \star S(A C)$

$A \star B \star E=C D S \triangle A \star B \star E+C S \triangle A \star B \star D \star E+D \triangle B \star E \star S(A C)+\triangle B \star D \star E \star S(A C)$

$C \star E=A B D S \triangle C \star E+A B S \triangle C A D \star E+B D \triangle E \star S(A C)+B A D \star E \star S(A C)$

$A \star C \star E=E D S \triangle A \star C \wedge E+B S \triangle A \star C \star D \star E+B D \Delta E \star S(A C)+B \wedge D \star E \star S(A C)$

$B \star C * E=A D S \triangle B * C * E+A S \triangle B \star C \star D * E+D \triangle B * E * S(A C)+\triangle B \star D \star E * S(A C)$

$A \star B \star C \star E=D S \triangle A \star B \star C \star E+S \triangle A \star B \star C \star D \star E+D A B \star E \star S(A C)+\triangle B \star D \star E \star S(A C)$

$D * E=A B C S \triangle D A E+B \triangle D A E A S(A C)$

$A \star D \star E=B C S \triangle A \star D \star E+B A D \star E \star S(A C)$

$B \star D * E=A C S \triangle B * D A E+\triangle B \star D \star E \star S(A C)$

$A \star B \star D \star E=C S \triangle A \star B * D * E+\triangle B * D A E \star S(A C)$

$C * D \star E=A B S \triangle C * D * E+B \triangle D * E * S(A C)$

$A \star C \star D \star E=B S \triangle A \star C * D \star E+B \triangle D \star E \star S(A C)$

$B * C * D * E=A S \wedge B \star C * D * E+\triangle B * D * E \star S(A C)$

$A \star B \star C * D * E=S \triangle A \star B * C * D * E+\triangle B * D * E \star S(A C)$

$S(A C)=E D E \triangle S(A C)+B E \triangle D+S(A C)$

$B * S(A C)=D E \Delta B * S(A C)+E \triangle B * D * S(A C)$

$D * S(A C)=B E A D * S(A C)$

$B * D * S(A C)=E \triangle B * D * S(A C)$

$E \star S(A C)=B D A E * S(A C)+B a D * E \star S(A C)$

$B \star E \star S(A C)=D \triangle B \star E \star S(A C)+\triangle B * D * E \star S(A C)$

$D k E \star S(A C)=B \Delta D * E \star S(A C)$

$E \star D \star E \star S(A C)=\triangle B \star D * E \star S(A C)$

Step 9: Variable output (Test statements)

TST

TEST $H=D \quad E=D \not S(A C)$

TEST $H=A \star D \quad E=D \star S(A C)$

TEST $H=B * D \quad E=B * D * S(A C)$

TEST $H=A \star B \star D \quad E=B \star D \star S(A C)$

TEST $H=C \star D \quad E=D * S(A C)$

TEST $H=A \wedge C A D \quad E=D * S(A C)$

TEST $H=B \star C * D \quad E=B * D * S(A C)$

TEST $H=A \star B \star C \star D \quad E=B \star D * S(A C)$ 
Table 3 Continued

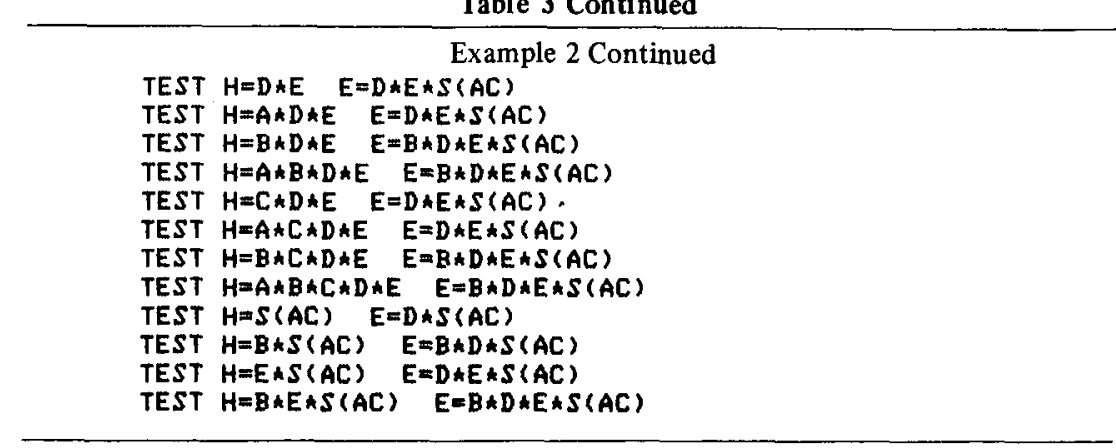

four parts to the output: (1) independent variables (stored under variable FRE), (2) model equation, (3) E(MS), and (4) test statements (see examples in Table 2).

Function 6: AID. The auxiliary function AID makes it possible to type a character vector that exceeds the length of the typing line (e.g., IBM 3767, 2741). For each line that exceeds the typing line, type the APL specification character $(\leftarrow)$ at the close of the line. Each line terminating with the specification character is catenated (less the specification character) to the existing character vector. The vector, once formed, is stored under Variable A. It should be noted that responses to Qs in function INPUT are formed by AID. For illustrations of the use of function AID, see Example 2 in Table 3.

Function 7: CMTX. The auxiliary function CMTX is designed to convert a character vector (row) into matrix form (design information is evaluated by rows). The number of rows equals the number of terms; the number of columns, the length of the longest term. Function CMTX was written by Gilman and Rose (1976, p. 153); only the name of the function (ROWNAMES) and certain variable names were changed.

Computer. All functions, with the possible exception of CMTX, were developed on an IBM 370/168 under $\mathrm{APL} / \mathrm{SV}$ and are compatible under VS APL.
Availability. Additional information is available from the author.

\section{REFERENCE NOTE}

1. Barr, J., Goodnight, J. H., Sall, J. P., \& Helwig, J. T. $A$ user's guide to SAS 76. North Carolina: SAS Institute, 1976.

\section{REFERENCES}

Bennett, C. A., \& Franklin, N. L. Statistical analysis in chemistry and the chemical industry. New York: John Wiley, 1954.

Cornfield, J., \& Tukey, J. W. Average values of mean squares in factorials. Annals of Mathematical Statistics, 1956, 27, 907-949.

Gilman, L., \& Rose, A. J. APL: An interactive approach (2nd ed.). New York: John Wiley, 1976.

KIRK, R. E. Experimental design: Procedures for the behavioral sciences. Belmont, Calif: Brooks/Cole, 1968.

WINER, B. J. Statistical principles in experimental design (2nd ed.). New York: McGraw-Hill, 1971.

\section{NOTE}

1. For those not familiar with the terminology employed, the reader is referred to Kirk (1968).

(Accepted for publication April 10, 1978.) 